\title{
Schülervorstellungen in einem inquiry-orientierten Sachunterricht verändern - Besteht ein Zusammenhang mit der Förderung adäquaten Schlussfolgerns?
}

\author{
Hanna Grimm ${ }^{1}$ (D) Maria Todorova ${ }^{1}$ Kornelia Möller ${ }^{1}$ \\ Eingegangen: 24. Mai 2019/ Angenommen: 28. Februar 2020 / Online publiziert: 11. März 2020 \\ (c) Der/die Autor(en) 2020
}

\section{Zusammenfassung}

Die Fähigkeit des hypothesenbezogenen Schlussfolgerns wird in der aktuellen Forschung als ein relevanter Faktor behandelt, der im Rahmen der Hypothesenprüfung im inquiry-orientierten Unterricht zur Erkennung von diskrepanten Ereignissen beiträgt und somit einen kognitiven Konflikt beim Lernenden begünstigen kann. Die Unzufriedenheit eines Lernenden mit den eigenen alternativen Vorstellungen gilt als förderlich für deren Abschwächung und für den Aufbau des wissenschaftlich adäquaten Konzepts. Der Zusammenhang zwischen dem hypothesenbezogenen Schlussfolgern und alternativen Vorstellungen ist bislang kaum untersucht worden. In der vorliegenden Interventionsstudie wurde ein inquiry-orientierter Unterricht zum Thema „Wie kommt es, dass ein Ball springt?“ durchgeführt ( $N=143$ Drittklässler/innen). Während in einer Kontrollgruppe ein Unterricht zur Unterstützung von Schülervorstellungsveränderungen stattfand, erhielt eine Experimentalgruppe denselben Unterricht mit zusätzlicher Förderung des hypothesenbezogenen Schlussfolgerns. In einem Prä-Post-Follow-Up-Design wurden sowohl das hypothesenbezogene Schlussfolgern als auch die alternativen Vorstellungen zum Sprungverhalten von Gegenständen erfasst, um die Veränderung der alternativen Vorstellungen über die Zeit und den Zusammenhang zwischen dem hypothesenbezogenen Schlussfolgern und alternativen Vorstellungen mithilfe von Mehrebenenanalysen zu untersuchen. Zudem wurde überprüft, inwiefern sich die zusätzliche Förderung des hypothesenbezogenen Schlussfolgerns in der Experimentalgruppe auf den Entwicklungsverlauf der alternativen Vorstellungen auswirkt. Die Ergebnisse zeigen für die Gesamtkohorte eine signifikante hypothesenkonforme Abschwächung alternativer Vorstellungen nach dem Unterricht und eine erneute geringfügige Zunahme dieser nach einigen Monaten. Zudem geht eine höhere Ausprägung der Fähigkeit, widerlegende und irrelevante Ereignisse bei der Überprüfung von konditionalen Aussagen adäquat zu evaluieren, mit einer signifikant geringeren Zustimmung zu alternativen Vorstellungen einher. Darüber hinaus liegt eine signifikante Cross-Level-Interaktion zwischen der Interventionsgruppe und dem Entwicklungsverlauf der alternativen Vorstellungen zugunsten der Experimentalgruppe vor. Die Ergebnisse deuten darauf hin, dass die Schlussfolgerungsfähigkeit und deren Förderung im naturwissenschaftlichen Unterricht einen positiven Einfluss auf die Veränderung von alternativen Vorstellungen haben kann.

Schlüsselwörter hypothesenbezogenes Schlussfolgern · Schülervorstellungen · naturwissenschaftlicher Sachunterricht · scientific inquiry

Hanna Grimm

hanna.grimm@uni-muenster.de

1 Institut für Didaktik des Sachunterrichts, Westfälische Wilhelms-Universität Münster, Leonardo-Campus 11, 48149 Münster, Deutschland 


\title{
Changes in Students' Conceptions in Inquiry-Oriented Primary Science Classes-Is There a Connection to the Promotion of Logical Reasoning?
}

\begin{abstract}
In current research, the ability to use hypothesis-based reasoning is treated as a relevant factor that contributes to the successful detection of inconsistent results during hypothesis testing in inquiry-based instruction and thus helps to promote cognitive conflict in the learner. The learner's dissatisfaction with their own alternative conceptions is considered conducive to the weakening of such notions, and aides the development of a more scientifically appropriate concept. The relationship between the hypothesis-based reasoning and students' alternative conceptions has hardly been studied so far. In the present study, an inquiry-based instruction to promote the development of hypothesis-based reasoning in primary science was conducted on the topic, "How come a ball bounces?" ( $N=143$ third graders). While in a control condition lessons took place to support conceptual change, an experimental condition received the same lessons with additional promotion of hypothesis-based reasoning. In a pretest-posttest design with follow-up, hypothesis-based reasoning and alternative conceptions of the bouncing behavior of objects were recorded with the goal of investigating changes in students' alternative conceptions about time and the relation between reasoning and alternative conceptions using a multilevel-analysis approach. Additionally, the impact of the intervention condition on the development of alternative conceptions was investigated. Results for both groups reveal a significant weakening of alternative conceptions following instruction, which was consistent with our hypothesis, and a renewed slight increase after a few months. In addition, a higher ability to evaluate refuting or irrelevant evidence through the testing of conditional statements is associated with a lower validation of alternative conceptions. Besides, it exists a significant cross-level-interaction between the intervention condition and the development of alternative conceptions in favor of the experimental condition. The results suggest that promoting reasoning ability can have a positive impact on changing students' conceptions in science education.
\end{abstract}

Keywords hypothesis-based reasoning $\cdot$ prior conceptions $\cdot$ primary science teaching $\cdot$ scientific inquiry

\section{Einleitung}

Als mittlerweile anerkannt gilt, dass im naturwissenschaftlichen Unterricht sowohl prozess- als auch inhaltsbezogene Kompetenzen zu fördern sind (Bybee 2002); dieser Anspruch trifft auch bereits auf den Sachunterricht in der Grundschule zu (GDSU 2013; Prenzel et al. 2007). Um beide Kompetenzen parallel in den Blick zu nehmen, eignet sich ein Unterricht, in dem die Schülerinnen und Schüler (SuS) ihre eigenen Vorstellungen auf ihre Gültigkeit hin experimentell überprüfen. In solch einem Unterricht, häufig als scientific inquiry-orientiert bezeichnet, erwerben und verwenden die SuS verschiedene naturwissenschaftliche Denk- und Arbeitsweisen. Die Förderung solcher prozessbezogenen Kompetenzen wiederum hat sich in zahlreichen Studien als förderlich für konzeptuelles Lernen erwiesen (Alfieri et al. 2011; Decristan et al. 2015; Edelsbrunner et al. 2018; Furtak et al. 2012; Minner et al. 2010). Eine zentrale prozessbezogene Kompetenz in den Naturwissenschaften ist das Ziehen von hypothesenbezogenen Schlussfolgerungen aus Experimenten (Prenzel et al. 2007). Es kann angenommen werden, dass auch dieser Kompetenz eine wichtige Rolle beim inhaltsbezogenen Lernen zukommt.

Kinder kommen mit mehr oder weniger tief verankerten Erklärungen und Vorstellungen zu Phänomenen aus ihrem Alltag in den Unterricht. Vorstellungen, die von den wissenschaftlich anerkannten Konzepten abweichen, werden in der Literatur als alternative Vorstellungen bezeichnet (Gropengießer und Marohn 2018; Wandersee et al. 1994) ${ }^{1}$. Die Veränderung von alternativen Vorstellungen gilt im Rahmen der Conceptual Change-Forschung als ein wichtiger Schritt, um wissenschaftlich adäquatere Konzepte aufbauen zu können (Limón 2001; Posner et al. 1982). Eine Veränderung kann anhand verschiedener Strategien angestrebt werden wie z.B. mit der Anknüpfungs- oder der Brückenstrategie. Bei der Anknüpfungsstrategie wird auf vorhandenen Vorstellungen aufgebaut und diese werden weiter differenziert, während bei der Brückenstrategie auf das Bewusstmachen der vorhandenen Vorstellungen zu Beginn des Unterrichts verzichtet wird. Stattdessen werden bei dieser Strategie die Ausgangsvorstellungen erst nach der Erarbeitung angemessener Vorstellungen reflektiert (Scott et al. 1992). Eine weitere Strategie beruht darauf, als Ausgangspunkt für die Veränderung alternativer Vorstellungen im Unterricht einen kognitiven Konflikt hervorzurufen, welcher darauf abzielt, alternative Vorstellungen durch die Konfrontation mit einem widerlegenden Ereignis zu erschüttern (Limón

\footnotetext{
${ }^{1}$ Zur Bezeichnung von Schülervorstellungen, die nicht mit wissenschaftlich anerkannten Konzepten übereinstimmen, werden eine Reihe weiterer Begriffe verwendet, z.B. Fehlvorstellungen, Fehlkonzepte, Misskonzepte und naive Vorstellungen. Die Bezeichnung alternative Vorstellungen wird in Anlehnung an Gropengießer und Marohn (2018) in diesem Beitrag als neutrale und respektvolle Bezeichnung verwendet.
} 
2001). Die empirische Befundlage zur Wirksamkeit dieser sog. Konfliktstrategie zur Veränderung alternativer Vorstellungen ist jedoch nicht eindeutig (Limón 2001; Ohlsson 2009; Ramsburg und Ohlsson 2016). Widerlegende Information führt bspw. nicht immer zu einem bedeutsamen kognitiven Konflikt beim Lernenden; besonders bei der Überprüfung von tief verankerten alternativen Vorstellungen treten häufig Schwierigkeiten bezüglich der Anwendung adäquater Schlussfolgerungen auf (Chinn und Brewer 1993, 1998).

Bei der experimentellen Überprüfung von alternativen Vorstellungen ist es erforderlich, einen Rückschluss vom beobachteten Ereignis des Experiments auf die anfänglich formulierte Vermutung zu ziehen und zu entscheiden, ob sich die Vorstellung als haltbar erweist oder nicht. Nur durch das Erkennen von widerlegender Evidenz kann die alternative Vorstellung als nicht haltbar erkannt und in ihrer Gültigkeit erschüttert werden, sodass in diesem Kontext adäquates hypothesenbezogenes Schlussfolgern für die Veränderung von alternativen Vorstellungen zentral erscheint.

Hypothesenbezogene Schlussfolgerungen stellen allerdings insbesondere für Grundschulkinder, aber auch noch für ältere SuS sowie Erwachsene, eine Herausforderung dar (Gauffroy und Barrouillet 2011). Somit besteht für den naturwissenschaftlichen Unterricht der Grundschule in zweifacher Hinsicht der Bedarf einer Förderung des hypothesenbezogenen Schlussfolgerns - zum einen als prozessbezogene Kompetenz und zum anderen als mögliche Voraussetzung für die Veränderung von alternativen Vorstellungen.

Die Möglichkeit einer Förderung des hypothesenbezogenen Schlussfolgerns bei Grundschulkindern konnte bereits in vorangegangenen Studien gezeigt werden (Robisch et al. 2014; Grimm et al. 2018). Die Veränderung alternativer Vorstellungen im Rahmen solcher Fördersettings sowie deren Zusammenhang mit der Fähigkeit zum hypothesenbezogenen Schlussfolgern ist jedoch bislang noch kaum untersucht. Ergebnisse aus Studien mit computerbasierten Instruktionen im Bereich der Sekundarstufe deuten darauf hin, dass eine höhere Schlussfolgerungsfähigkeit mit der Veränderung alternativer Vorstellungen einhergeht (Liao und She 2009; Kang et al. 2004). Inwiefern der Zusammenhang zwischen hypothesenbezogenem Schlussfolgern und alternativen Vorstellungen für einen inquiry-orientierten Unterricht im Grundschulbereich aufgezeigt werden kann, ist unklar. Daher verfolgt die vorliegende Studie drei Ziele:

1. die Veränderung alternativer Vorstellungen bei Grundschulkindern über die Zeit zu untersuchen,

2. den möglichen Zusammenhang zwischen der Fähigkeit zum hypothesenbezogenen Schlussfolgern und alternativen Vorstellungen bei Grundschulkindern zu überprüfen sowie
3. den Effekt einer zusätzlichen Förderung des hypothesenbezogenen Schlussfolgerns auf den Entwicklungsverlauf der alternativen Vorstellungen festzustellen.

\section{Entwicklung der Fähigkeit des hypothesenbezogenen Schlussfolgerns}

Hypothesen treten oftmals in der Form von konditionalen Aussagen auf, wie zum Beispiel ,Wenn ein Ball rund ist, dann springt er'. Bei der Überprüfung von konditionalen Aussagen der Form ,wenn $p$, dann $q^{6}$, gilt es zwischen vier möglichen kombinatorischen Ereignissen von $p$ und $q \mathrm{zu}$ unterscheiden und das vorliegende Ereignis adäquat zu beurteilen. Das Ereignis $p q$ [rund, springt] bekräftigt die Hypothese, das Ereignis $p \neg q$ [rund, springt nicht] widerlegt die Hypothese. Während anhand dieser beiden Ereignisse eine Aussage über den Wahrheitsgehalt der Hypothese getroffen werden kann, bieten die beiden Ereignisse $\neg p q$ und $\neg p \neg q$ [jeweils nicht rund] keinen Informationsgehalt in Bezug auf die Hypothese (Lawson 2009). Es sind daher für die Überprüfung der Hypothese irrelevante Ereignisse. In Anlehnung an den kognitionspsychologischen Terminus ,reasoning about truth-values' (Barrouillet et al. 2008) wird im Folgenden der Begriff ,hypothesenbezogenes Schlussfolgern ' im Kontext der Evaluation von konditionalen Aussagen verwendet.

Sowohl in kognitionspsychologischen (Barrouillet et al. 2008; Gauffroy und Barrouillet 2011) als auch in fachdidaktischen Untersuchungen (Robisch et al. 2014; Tröbst et al. 2011) hat sich gezeigt, dass SuS im Grundschulalter, aber auch noch darüber hinaus, häufig Probleme beim hypothesenbezogenen Schlussfolgern aufweisen. Insbesondere das Erkennen von irrelevanten Ereignissen stellt für Kinder im Grundschulalter eine Herausforderung dar. Erst bei SuS der neunten Klasse zeigt sich ein gesteigertes Verständnis bei der Evaluation irrelevanter Ereignisse, welches jedoch nach wie vor nicht voll ausgeprägt ist. Erst im Erwachsenenalter werden hypothesenbezogene Schlussfolgerungen in Bezug auf alle vier Ereignisevaluationen vornehmlich adäquat evaluiert (Gauffroy und Barrouillet 2011). Als Einflussfaktoren auf die Entwicklung von hypothesenbezogenen Schlussfolgerungen gelten die Fähigkeit zur Inhibition spontaner Reize zugunsten analytischer Prozesse (Handley et al. 2004) sowie die Arbeitsspeicherkapazität (Klauer et al. 1997).

Da Grundschulkindern adäquate Schlussfolgerungen bei der Überprüfung von konditionalen Aussagen häufig nicht gelingen, hypothesengeleitetes Experimentieren jedoch bereits in der Grundschule zu den Inhalten einer naturwissenschaftlichen Grundbildung gehört (Prenzel et al. 2007), erscheint eine Förderung des hypothesenbezogenen Schlussfolgerns im Sachunterricht sinnvoll. Vorangegangene Studien konnten bereits die Möglichkeit einer Förderung des 
hypothesenbezogenen Schlussfolgerns im Grundschulalter durch den Einsatz von sog. Scaffolding-Maßnahmen im Einzelsetting unter Laborbedingungen (Robisch et al. 2014; Tröbst et al. 2011) und in ganzen Schulklassen (Grimm et al. 2018) aufzeigen. Scaffolding beschreibt eine interaktive Form der Lernunterstützung, die einerseits Lerninhalte so strukturiert, dass sie für den Lerner erfassbar gemacht werden, und andererseits zur Förderung einer elaborierten mentalen Auseinandersetzung kognitiv aktivierende Lernimpulse setzt. Indem die Unterstützung angepasst an den Lernstand des Lernenden schrittweise zurückgenommen wird, steigt die Verantwortung für den Lernprozess auf Seiten des Lernenden (Kleickmann at al. 2012; van de Pol et al. 2010). In der Studie mit ganzen Schulklassen wurde ein scientific inquiry-orientierter Unterricht in zwei Gruppen durchgeführt, der die Überprüfung von Schülervorstellungen in Form von konditionalen Aussagen beinhaltete. In einer Experimentalgruppe (EG) wurden im Vergleich zu einer Kontrollgruppe (KG) zusätzlich Scaffoldingmaßnahmen zur Förderung des hypothesenbezogenen Schlussfolgerns eingesetzt. Während der Unterricht in beiden Gruppen zu einer Verbesserung des hypothesenbezogenen Schlussfolgerns führte, zeigte sich in der EG ein signifikant höherer Lernzuwachs (Grimm et al. 2018). Die Daten aus dieser Studie werden auch zur Untersuchung der diesem Beitrag zugrundeliegenden Fragestellungen verwendet.

\section{Entstehung und Entwicklung von alternativen Vorstellungen}

Bevor SuS in den Unterricht eintreten, haben sie bereits im Alltag zu diversen naturwissenschaftlichen Themen Erfahrungen gesammelt und Vorstellungen konstruiert. Häufig weichen die Vorstellungen der SuS allerdings von den als wissenschaftlich adäquat geltenden Konzepten ab (Adamina et al. 2018; Duit 1995; Duit und Treagust 2003; Möller 2018; Vosniadou et al. 2001). Die Entstehung von alternativen Vorstellungen im Alltag kann auf unterschiedliche Art und Weise erfolgen, wie zum Beispiel durch das Interpretieren von Alltagserfahrungen (z.B. Kleidung aus Wolle wärmt, also erzeugt Wolle Wärme) oder durch missverständliche alltagssprachliche Formulierungen (z.B. der Strom wird verbraucht oder morgens geht die Sonne auf) (Möller 2019).

Beeinflusst durch ihre Entstehung können Vorstellungen beim Lernenden unterschiedlich tief verankert sein. Vorstellungen, die sich im Alltag bereits in vielen Situationen bewährt haben, sind meist tief verwurzelt und haben eine hohe Glaubwürdigkeit für die Lernenden. Solche stabilen Überzeugungen oder auch sog. deep structures basieren auf der Einbindung in Schemata (Niedderer und Schecker 1992), weshalb sie häufig resistent gegen Veränderungen sind (Adamina et al. 2018; Duit 1995) und den Lernprozess erschweren können (Vosniadou et al. 2001; Wodzinski 2006). Dies zeigt sich beispielsweise daran, dass alternative Vorstellungen häufig auch parallel zu dem neu erworbenen wissenschaftlichen Konzept weiterbestehen (Caravita und Halldén 1994; Limón 2001). Dies ist kaum verwunderlich, da sich die im Alltag konstruierten Konzepte auch weiterhin in der Lebenswelt der Kinder bewähren (Carey 2000; Smith et al. 1993). Zum Beispiel antworten Kinder auf die Frage „Wie kommt es, dass ein Ball springt?“ häufig, dass das Springen an der im Ball vorhandenen Luft liegt. Diese Vorstellung hat sich über vielfache Erfahrung aufgebaut, z.B. beim Erleben davon, dass platte Bälle weniger gut springen. Diese zwar nicht falsche, aber eben nicht umfassend wissenschaftliche Erklärung, bleibt durch die langfristige Bewährung im Alltag häufig parallel zum im Unterricht erarbeiteten wissenschaftlichen Konzept bestehen. Weitere häufig genannte Vorstellungen von SuS im Grundschulalter zu der Frage „Wie kommt es, dass manche Dinge springen und andere Dinge nicht?" beziehen sich auf die Form (runde Dinge springen; eckige Dinge springen nicht), den Härtegrad (harte Dinge springen; weiche Dinge springen nicht) und das Gewicht von Gegenständen (leichte Dinge springen; schwere Dinge springen nicht) (Möller 2019).

Neben tief verwurzelten Vorstellungen gibt es Schülervorstellungen, die auf Basis von Ad-hoc-Konstruktionen entstehen und als sog. current constructions bezeichnet werden (Niedderer und Schecker 1995). Current constructions können zum Beispiel entstehen, wenn SuS nach ihren vorunterrichtlichen Vorstellungen befragt werden und keine deep structures vorhanden sind. Ihre Veränderung fällt den Lernenden in der Regel leichter (Möller 2019).

Eine zentrale Strategie, um im Unterricht Vorstellungsveränderungen zu initiieren, ist der auf Posner et al. (1982) zurückgehende Ansatz der Konfliktstrategie (Lin et al. 2016). Die Konfliktstrategie beruht auf der Annahme, dass das Eintreten von Unzufriedenheit mit der vorhandenen Vorstellung eine von vier Bedingungen für konzeptuelle Veränderungen darstellt (Posner et al. 1982). Der Zusammenhang zwischen der Erzeugung eines kognitiven Konflikts durch die Präsentation von widersprüchlichen Ereignissen und der Veränderung von alternativen Vorstellungen konnte bereits in einigen Studien gezeigt werden. Kang et al. (2004) sowie Lee und Byun (2012) führten Interventionsstudien mit Sekundarschülerinnen und -schülern durch, indem sie zunächst die Vorstellungen der SuS erhoben und daraufhin die Lernenden mit einem widerlegenden Ereignis konfrontierten. Kang et al. (2004) operationalisierten das Erleben eines kognitiven Konflikts über die Antworttypen bei der Reaktion auf ein widerlegendes Ereignis nach Chinn und Brewer (1998) und führten eine Prä-Post Erhebung zu den Schülervorstellungen im Themenbereich Dichte durch. Es zeigte sich eine signifikante Korrelation 
zwischen dem Grad des kognitiven Konflikts und den Vorstellungsveränderungen. Lee und Byun (2012) erfassten den kognitiven Konflikt über Schüleraussagen zum Erleben des widerlegenden Ereignisses im Themenfeld Mechanik und operationalisierten die Vorstellungsveränderung über eine Einordnung von Schüleraussagen in die Antworttypen von Chinn und Brewer (1998). In dieser Untersuchung zeigte sich im Einklang mit der Studie von Kang et al. (2004), dass bei stärkerem Erleben eines kognitiven Konflikts häufiger Vorstellungsveränderungen eintraten.

Die beiden Studien von Kang et al. (2004) und Lee und Byun (2012) implizieren, dass Vorstellungsveränderungen abrupte Vorgänge beim Erleben eines einzigen Ereignisses sind. Die Conceptual Change-Forschung weist jedoch auch Befunde vor, die zeigen, dass bei Vorstellungsveränderungen kein plötzlicher Wechsel stattfindet, sondern Vorstellungsveränderungen vielmehr in einem graduellen Prozess der Restrukturierung verlaufen (Caravita 2001; Vosniadou et al. 2001). Die erforderliche Zeit für solche Umstrukturierungsprozesse ist allerdings im Kontext des Schulunterrichts nicht immer gegeben. Wenn daher vollständige oder endgültige Vorstellungsveränderungen im Unterricht nicht gelingen, so könnten jedoch der Grad an Überzeugung und die Reichweite der alternativen Vorstellungen zurückgehen. Bei der Vorstellung, dass luftgefüllte Dinge springen, kann so möglicherweise beim Lernenden die Einsicht erreicht werden, dass das Luftkonzept nicht in allen Fällen als Erklärung dienen kann.

Neben dem Befund, dass Vorstellungsveränderungen einen langwierigen Prozess durchlaufen, hat sich in der Conceptual Change-Forschung auch gezeigt, dass alternative Vorstellungen nach stattgefundener Instruktion durchaus wieder zurückkehren können. In der Interventionsstudie von Hardy et al. (2006) zur Förderung von konzeptuellem Lernen beim Thema Dichte und Auftrieb mit SuS der dritten Klasse zeigte sich in einem Follow Up-Test nach einem Jahr, dass SuS, die ein höheres Ausmaß an Lernunterstützung erhielten, ihr Level des konzeptuellen Verständnisses aufrechterhalten konnten. Bei SuS hingegen, die ein geringeres Ausmaß an Lernunterstützung erhielten, traten die alternativen Vorstellungen ein Jahr nach dem Unterricht wieder auf. Die Studie liefert Hinweise darauf, dass der Einsatz von Lernunterstützung in Form von Elementen der Strukturierung und des Problematisierens ein Faktor sein kann, der langfristige Vorstellungsveränderungen im Unterricht positiv beeinflussen kann. Es gibt zahlreiche weitere empirische Evidenz, die zeigt, dass Scaffolding das Lernen bzw. die Integration von neuem Wissen in vorhandene Wissensstrukturen positiv beeinflusst (Decristan et al. 2015; van de Pol et al. 2010; Wittwer und Renkl 2008). Vorstellungsveränderungen werden in der Conceptual Change-Theorie des Kohärenzansatzes (Vosniadou und Brewer 1992) als Integration von neuem Wissen in vorhan- dene Wissensstrukturen beschrieben. Durch den Aufbau von sogenannten synthetischen Modellen entsteht für den Lerner ein subjektiv konsistentes Erklärungssystem. Kann neues Wissen jedoch nicht in bestehende Erklärungssysteme integriert werden, stehen wissenschaftlich korrekte Informationen unverbunden neben den alternativen Vorstellungen (Hardy und Meschede 2018). Der Einsatz von Scaffolding scheint dem parallelen Bestehen von alternativen und wissenschaftlichen Vorstellungen entgegenwirken zu können.

Neben lernunterstützenden Maßnahmen hat die Forschung zu Vorstellungsveränderungen auch kognitive Faktoren, wie das schlussfolgernde Denken identifiziert, die den Erfolg von Konzeptveränderungen im Unterricht beeinflussen können (Kang et al. 2010; Leonard et al. 2015; Lin et al. 2016).

\section{Zusammenhang zwischen der Schluss- folgerungsfähigkeit und alternativen Vorstellungen}

Die Untersuchung von Einflussfaktoren auf die Veränderung von Schülervorstellungen ist Gegenstand zahlreicher Studien in der Conceptual Change-Forschung. Lin et al. (2016) zeigen in ihrem Review von 116 Studien auf, dass nach dem Einfluss von instruktionalen Maßnahmen auf Vorstellungsveränderungen am häufigsten die Schlussfolgerungsfähigkeit als Prädiktor für Conceptual ChangeProzesse untersucht wurde. Lawson (2003) begründet die Annahme eines Zusammenhangs zwischen der Veränderung alternativer Vorstellungen und der Schlussfolgerungsfähigkeit von Lernenden damit, dass logisches Denken erforderlich ist, um Evidenz adäquat in Bezug zur eigenen Vorstellung zu setzen. Lawson und Weser (1990) zeigten in einer Studie mit einem Prä-Post-Design, dass Universitätsstudierende im Fach Biologie mit geringerer Schlussfolgerungsfähigkeit weniger bereit waren, ihre nicht wissenschaftlichen Vorstellungen in Folge einer Intervention aufzugeben. Die Schlussfolgerungsfähigkeit wurde anhand des Scientific Reasoning Tests (SRT) von Lawson (1978) gemessen, welcher eine multiple Perspektive auf das logische Denken abbildet.

Ähnliche Ergebnisse konnten im schulischen Kontext für die Sekundarstufe aufgezeigt werden. Kang et al. (2004) führten mit SuS der siebten Klasse eine 15-minütige computerbasierte Instruktion zum Thema Dichte durch. Die Schülervorstellungen wurden vor und nach der Instruktion erhoben, während das logische Denken, erfasst über die vergleichbaren Komponenten des SRT, einmalig vor der Intervention erfasst wurde. Während die SuS mit gering ausgeprägtem logischen Denken eher an ihren alternativen Vorstellungen festhielten, akzeptierten $\mathrm{SuS}$ mit hoch 
ausgeprägtem logischen Denken diskrepante Ereignisse als solche und zeigten sich eher unzufrieden mit vorhandenen alternativen Vorstellungen. $\mathrm{Zu}$ vergleichbaren Ergebnissen gelangten Liao und She (2009) und Lee und She (2010), in deren Studien computerbasierte Instruktionen mit einem zeitlich größeren Ausmaß von zehn bzw. acht Unterrichtsstunden durchgeführt wurden. Das digitale Lernprogramm zielte darauf $a b$, sowohl das schlussfolgernde Denken als auch das konzeptuelle Verständnis in den Themenbereichen Atome bzw. Verbrennung zu fördern. Auch in diesen beiden Studien wurden Prä-Post-Messungen zu den konzeptuellen Vorstellungen sowie der SRT durchgeführt. Der Vergleich von zwei Gruppen mit und ohne computerbasierter Instruktion zur Förderung des Schlussfolgerns und des konzeptuellen Verständnisses zeigte, dass SuS, die die Förderung erhielten, häufiger ihre alternativen Vorstellungen veränderten. Es zeigte sich ein Zusammenhang zwischen der Schlussfolgerungsfähigkeit und der Anzahl veränderter alternativer Vorstellungen. Diese Ergebnisse deuten darauf hin, dass eine Förderung des Schlussfolgerns wirksam für die Initiierung von Conceptual-Change-Prozessen sein könnte.

Für Kinder im Grundschulalter konnte im Projekt Science- $P$ anhand von Querschnittsdaten naturwissenschaftliches Denken als Prädiktor für konzeptuelles Verstehen identifiziert werden (Pollmeier et al. 2017). Die Schülervorstellungen in den Bereichen Schwimmen und Sinken sowie Verdunstung und Kondensation wurden mit einem Multiple Choice Test erhoben und drei Verständnisleveln zugeordnet. Das naturwissenschaftliche Denken umfasste die Operationalisierung von Experimentierstrategien, den Aufbau von schlüssigen Experimenten, das Interpretieren von Evidenz und des Wissenschaftsverständnisses.

Die vorliegenden Studien lassen annehmen, dass logisches Denken in einem Zusammenhang mit der Veränderung von Schülervorstellungen steht. Die bisherigen Studien operationalisieren die Schlussfolgerungsfähigkeit allerdings über ein breites Spektrum an logischen Denkoperationen, sodass der Zusammenhang nicht auf bestimmte Denkprozesse zurückgeführt werden kann. Es liegen bislang keine Studien vor, die den Fokus auf nur eine Facette des logischen Denkens, wie zum Beispiel das hypothesenbezogene Schlussfolgern bei konditionalen Aussagen, legen. Dabei sind Vermutungen zur Erklärung von naturwissenschaftlichen Phänomenen häufig in Form von konditionalen Aussagen formuliert und damit auch Gegenstand eines inquiry-orientierten Unterrichts. Da SuS oft nicht in der Lage sind, adäquate Ereignisevaluationen bei der Überprüfung von konditionalen Aussagen zu treffen (Gauffroy und Barrouillet 2011), ist es von Interesse zu untersuchen, inwiefern dieser Aspekt der Schlussfolgerungsfähigkeit in einem $\mathrm{Zu}-$ sammenhang mit alternativen Vorstellungen steht. Außerdem wurden bisher entweder Querschnittsstudien oder Stu- dien mit ausschließlich computerbasierten Interventionen durchgeführt. Es liegen keine Ergebnisse dazu vor, ob die Schlussfolgerungsfähigkeit mit der Zustimmung zu alternativen Vorstellungen in üblichen lehrkraftbasierten Unterrichtsinterventionen zusammenhängt. Darüber hinaus handelt es sich fast ausschließlich um Interventionen im Sekundarstufenbereich oder im universitären Kontext. Für Kinder im Grundschulalter gibt es bisher keine bekannten Untersuchungen, in denen neben dem Aufbau des konzeptuellen Verständnisses eine Förderung der Schlussfolgerungsfähigkeit berücksichtigt wird.

\section{Fragestellungen und Hypothesen}

Um diese Forschungslücke aufzugreifen, wurde in der vorliegenden quasi-experimentellen Studie mit einem Kontrollgruppendesign folgenden drei Fragestellungen nachgegangen:

FF 1 Wie entwickelt sich die Anzahl an alternativen Vorstellungen von Grundschulkindern zum Thema „Wie kommt es, dass ein Ball springt?" von vor dem Unterricht zu nach dem Unterricht bis hin zu 12-14 Wochen nach dem Unterricht?

Der Einsatz der Konfliktstrategie kann dazu führen, dass SuS die Überzeugung bzgl. ihrer alternativen Vorstellungen verlieren oder abschwächen. Häufig sind alternative Vorstellungen jedoch sehr resistent gegen Veränderungen und können auch noch einige Zeit nach einem Unterricht wieder zurückkehren (Limón 2001). In der Studie von Hardy et al. (2006) hat sich gezeigt, dass die Anzahl an alternativen Vorstellungen durch unterrichtliche Instruktion zunächst abnimmt, ein Jahr nach der Instruktion - abhängig vom Ausmaß an Strukturierung der Lerngelegenheit - entweder wieder steigt oder konstant bleibt. Hiervon ausgehend ist anzunehmen, dass es von Prä zu Post einen Rückgang an alternativen Vorstellungen geben wird, die zum Follow-Up-Zeitpunkt hin allerdings wieder ansteigen können.

FF 2 Besteht ein Zusammenhang zwischen dem hypothesenbezogenen Schlussfolgern und den alternativen Vorstellungen der SuS?

Ausgehend von der Annahme, dass die Schlussfolgerungsfähigkeit dazu beiträgt, Evidenz adäquat zu evaluieren und damit alternative Vorstellungen in ihrer Gültigkeit zu erschüttern (Kang et al. 2004), wird ein negativer Zusammenhang erwartet: Höhere Werte beim hypothesenbezogenen Schlussfolgern gehen mit einer geringeren Zustimmung zu alternativen Vorstellungen einher.

FF 3 Inwiefern bestehen Unterschiede im Entwicklungsverlauf der alternativen Vorstellungen zwischen einer Gruppe 
mit zusätzlicher Förderung und einer Gruppe ohne zusätzliche Förderung des hypothesenbezogenen Schlussfolgerns?

Es wird angenommen, dass sich ein Unterschied zwischen den Gruppen in der langfristigen Entwicklung der alternativen Vorstellungen nach dem Unterricht zeigen wird. Durch den Einsatz einer zusätzlichen Lernunterstützung in Form von inhaltlicher Strukturierung und kognitiver Aktivierung (Scaffolding) sollten integrative Lernprozesse mit einer langfristigen Wirkung begünstigt werden (Vosniadou und Brewer 1992). Eine Unterstützung der Fähigkeit des hypothesenbezogenen Schlussfolgerns könnte das Ablegen von alternativen Vorstellungen und damit auch die Bildung eines synthetischen Modells begünstigen, was dem parallelen Bestehen von alternativen und wissenschaftlichen Vorstellungen entgegenwirken sollte. Diese Annahme wird durch eine Studie von Hardy et al. (2006) gestützt, die zeigte, dass ein höheres Maß an Lernunterstützung vor allem den langfristigen Rückgang von alternativen Vorstellungen begünstigt. Des Weiteren stützt sich unsere Annahme auf die Studien von Liao und She (2009) und Lee und She (2010), die auf einen positiven Einfluss der Förderung des Schlussfolgerns auf die Veränderung von alternativen Vorstellungen hindeuten, allerdings ohne die Untersuchung einer langfristigen Entwicklung. Vor diesem Hintergrund kann die Annahme formuliert werden, dass der Einsatz einer zusätzlichen Förderung des hypothesenbezogenen Schlussfolgerns zu einer geringeren Rückkehr an alternativen Vorstellungen nach dem Unterricht führen sollte.

\section{Methode}

\section{Stichprobe, Intervention und Untersuchungsdesign}

An der Studie mit quasi-experimentellem Prä-Post-FollowUp-Kontrollgruppendesign haben insgesamt 143 Drittklässlerinnen und Drittklässler aus sechs Klassen an Schulen in Nordrhein-Westfalen mit einem Durchschnittsalter von 9,11 Jahren $(S D=0,50)$ teilgenommen, davon 81 Mädchen und 62 Jungen. Alle sechs Klassen erhielten einen inquiryorientierten Unterricht mit dem gleichen zeitlichen Umfang, wobei in einer EG $(n=73)$ im Vergleich $\mathrm{zu}$ einer KG $(n=70)$ zusätzlich explizite Scaffoldingmaßnahmen zur Förderung des hypothesenbezogenen Schlussfolgerns eingesetzt wurden. Der Unterricht wurde von drei erfahrenen, speziell geschulten Sachunterrichtslehrkräften (die durchschnittliche Berufserfahrung der Lehrerinnen beträgt 19 Jahre, $S D=3,6$ ) in jeweils einer Schulklasse der EG und der $\mathrm{KG}$ durchgeführt, um personenbezogene Effekte auszubalancieren. Die thematische Fragestellung des Unterrichts lautete ,Wie kommt es, dass ein Ding springt oder nicht springt?" (vgl. Thiel 1987); sie wurde innerhalb von sieben Unterrichtsstunden á 45 min bearbeitet. In der ersten
Unterrichtsstunde wurde ausgehend von einem Impuls mit den SuS die Fragestellung formuliert. In der zweiten bis zur vierten Unterrichtsstunde stellten die SuS Vermutungen zu der Fragestellung auf und überprüften diese im Anschluss, indem sie zur Verfügung stehende Experimentiermaterialien auf ihr Sprungverhalten hin testeten. Hierzu mussten die SuS hypothesenbezogene Schlussfolgerungen anstellen. Die vorab gemeinsam formulierten alternativen Vorstellungen der SuS wurden mit einem Gegenbeispiel widerlegt und damit als nicht allumfassende Erklärungen herausgestellt. Die abschließenden drei Unterrichtsstunden dienten vorrangig dem Aufbau des wissenschaftlich anerkannten Konzepts. Die zusätzliche Förderung des hypothesenbezogenen Schlussfolgerns in der EG durch zusätzliche Scaffoldingmaßnahmen wurde dementsprechend in drei der sieben Unterrichtsstunden implementiert; sie fand in der zweiten bis vierten Unterrichtsstunde statt.

Der Einsatz dieser Maßnahmen bezweckte die Inhibition spontaner Reaktionen sowie eine Entlastung der Arbeitsspeicherkapazität. Hierzu wurden sowohl kognitiv aktivierende als auch strukturierende Scaffolds bei der Unterstützung von Schlussfolgerungsprozessen eingesetzt. Eine ausführliche Darstellung der in der EG eingesetzten Scaffoldingmaßnahmen ist bei Grimm et al. (2018) zu finden.

Die beiden Testinstrumente zur Messung des hypothesenbezogenen Schlussfolgerns und der alternativen Vorstellungen wurden zu drei Messzeitpunkten (Prä, Post, FollowUp) eingesetzt. Die beiden Prätests erfolgten an zwei Terminen innerhalb von drei Wochen vor Beginn des Unterrichts, um die von den Testungen hervorgerufene kognitive Belastung möglichst gering zu halten. Der Posttest zum hypothesenbezogenen Schlussfolgern erfolgte unmittelbar nach Abschluss der vierten Sequenz, da zu diesem Zeitpunkt die zusätzliche Förderung des hypothesenbezogenen Schlussfolgerns in der EG abgeschlossen war. Der Posttest zur Erhebung der Zustimmung zu alternativen Vorstellungen wurde nach Abschluss der gesamten Unterrichtsreihe durchgeführt, damit die SuS die Gelegenheit hatten, ein adäquates Konzept aufzubauen. Die Follow-Up-Erhebungen fanden 12-14 Wochen nach Abschluss der Unterrichtsreihe statt, wiederum in jeder Klasse an zwei Terminen.

Zur Vorbereitung der Lehrpersonen für die Durchführung des Unterrichts fanden drei dreistündige Schulungen statt. Die Lehrpersonen wurden in den fachlichen Hintergrund eingeführt, der vorgeschlagene Unterrichtsverlauf wurde gemeinsam erarbeitet und die Unterstützungsmaßnahmen zur Förderung des hypothesenbezogenen Schlussfolgerns wurden trainiert. Ein mit den Lehrkräften gemeinsam erarbeitetes, detailliertes Skript für den Unterricht sowie sämtliche für den Unterricht benötigten Schriftund Experimentiermaterialien wurden den Lehrkräften zur Verfügung gestellt. 


\section{Testinstrumente}

\section{Schlussfolgerungstest}

Der Test zur Erfassung des hypothesenbezogenen Schlussfolgerns wurde als materialgestützte Klassenbefragung im paper-pencil-Format durchgeführt. In sog. „truth-testing tasks" in Anlehnung an Barrouillet et al. (2008) wurden den SuS vier Vermutungen in Form von Relativsätzen mit jeweils vier Ereignissen präsentiert (z. B. Tim glaubt „Dinge, die mit Luft gefüllt sind, springen.“, „Hier seht ihr einen Tennisball, welcher mit Luft gefüllt ist und springt. Was sagt euch der Tennisball über Tims Vermutung?"). Die vier Ereignisse bildeten jeweils die vier kombinatorischen Ereignismöglichkeiten ab und wurden bei der Präsentation in ihrer Reihenfolge variiert. Die SuS sollten bei jedem der insgesamt 16 Ereignisse entscheiden, ob die präsentierten Ereignisse die jeweilige Vermutung bekräftigen, widerlegen oder keine Aussage über den Wahrheitsgehalt der Vermutung machen. Zur Auswertung des Tests wurden für alle vier möglichen Ereignisevaluationen ( $p q, p \neg q, \neg p q, \neg p \neg q)$ jeweils alle richtig evaluierten Ereignisse addiert und in Bezug zum erreichbaren Maximalwert gesetzt, sodass die Darstellung der Ergebnisse als der prozentuale Anteil richtig evaluierter Ereignisse zu interpretieren ist. Die mental models theory (Gauffroy und Barrouillet 2009; JohnsonLaird und Byrne 2002) gibt Anlass zu der Annahme, dass das hypothesenbezogene Schlussfolgern am besten auf drei Dimensionen (bekräftigende, widerlegende und irrelevante Ereignisevaluationen) abgebildet wird. Um dies zu überprüfen, wurden konfirmatorische Faktorenanalysen in der Software Mplus Version 7.11 unter Verwendung des WLSMV-Schätzers für kategoriale Daten geschätzt. Es zeigte sich zuerst, dass zwei der vier $p q$-Items wegen Deckeneffekten von den Analysen ausgeschlossen werden müssen. Das angepasste Modell mit drei Faktoren für die Konditionale $p q, p \neg q$ und $\neg p q / \neg p \neg q$ wies einen guten Modellfit $(\chi 2(741)=861,28 ; p<0,001$; RMSEA $=0,03$; $\mathrm{CFI}=0,98)$ und keine auffälligen Residualkovarianzen oder Modifikationsindizes auf. Die konfirmatorischen Faktorenanalysen zeigten demnach, dass die Fähigkeit zum hypothesenbezogenen Schlussfolgern theoriekonform anhand dreier Indikatoren basierend auf der Leistung der SuS auf den $p q, p \neg q$ und $\neg p q / \neg p \neg q$-Aufgaben adäquat abgebildet werden kann, was als ein Hinweis für die Validität des Tests interpretiert werden kann. Die interne Konsistenz des Tests geschätzt nach Dunn et al. (2014) beträgt $\omega=0,90$ im Prätest und $\omega=0,82$ im Posttest.

\section{Test zur Erhebung alternativer Vorstellungen}

Der Test zur Erfassung von alternativen Vorstellungen erfolgte ebenfalls als Klassenbefragung im paper-pencil-For- mat. Den SuS wurden insgesamt acht typische alternative Vorstellungen von Kindern zu der Frage „Woran liegt es, dass ein Ding springt oder nicht springt?" präsentiert, woraufhin auf einer fünfstufigen Skala anzugeben war, wie stark der Vermutung zugestimmt wird (stimmt, stimmt eher, kann mich nicht entscheiden, stimmt eher nicht, stimmt nicht). Die Auswertung erfolgte anschließend dichotom, indem die beiden Antwortoptionen ,stimmt“ und ,stimmt eher" auf der Skala als Zustimmung zur präsentierten alternativen Vorstellung gewertet wurden. Mittels der dichotomen Auswertung wurden die Daten auf die wesentliche Aussage hin zusammengefasst, ob eine Zustimmung oder die angestrebte Ablehnung der präsentierten alternativen Vorstellung vorliegt. Die Darstellung der Ergebnisse ist darauf ausgerichtet, die Abnahme der Zustimmung zu den alternativen Vorstellungen darzustellen, um die Erschütterung der bei den SuS vorhandenen Vorstellungen zu erfassen. Wenn z.B. von acht präsentierten alternativen Vorstellungen im Prätest drei Vorstellungen zugestimmt wird und im Posttest nur noch zwei Vorstellungen, so kann angenommen werden, dass die Glaubwürdigkeit einer der alternativen Vorstellungen abgenommen hat.

\section{Statistische Analysen}

Zur Beantwortung der vorliegenden Fragestellungen wurden Mehrebenenanalysen (MEA) berechnet. Mehrebenenmodelle stellen eine Erweiterung der Regressionsanalyse dar, wenn Beobachtungen oder Messwerte ineinander verschachtelt (genestet) sind. Es handelt sich folglich um gruppierte Daten, bei denen eine sog. hierarchische Datenstruktur vorliegt. In querschnittlichen Untersuchungen sind z. B. SuS (Level 1) in Klassen (Level 2) eingebettet, die letzteren sind wiederum in Schulen (Level 3) geschachtelt. Bei der Anwendung von Mehrebenenanalysen bei längsschnittlichen Daten (Anderson 2012; Heck et al. 2014; Hox 2013) sind mehrere Messwerte bzw. Beobachtungen (Level 1) innerhalb von Personen (Level 2) geschachtelt. In sog. linearen gemischten (oder Random-Koeffizienten-)Modellen für längsschnittliche Daten fungiert die Zeit als Level 1-Variable. Die Person bzw. ihre Merkmale fungieren als Level 2-Variable. Änderungen in der abhängigen Variable werden in linearen 2-Level gemischten Modellen als eine Funktion der Zeit modelliert. Im sog. Random-Intercept-RandomSlope-Modell (s. Anderson 2012) werden feste Regressionseffekte (Fixed-Effekte) und Zufallseffekte (Random-Effekte) für die Zeitvariable einschließlich des Intercepts geschätzt. Die durchschnittliche, intraindividuelle Änderung über die Zeit wird durch den Fixed-Effekt der Zeitvariable repräsentiert und die Varianz des Random-Effekts dieser Variable zeigt das Ausmaß an interindividuellen Unterschieden in den intraindividuellen Änderungen an. Die Interpretation des (Random) Intercepts hängt von der Ko- 
dierung der Zeitvariable ab. Wird z. B. der erste Messzeitpunkt mit 0 kodiert, so stellen der Fixed-Effekt des Intercepts die durchschnittliche Ausprägung der abhängigen Variable und die Varianz die interindividuellen Unterschiede jeweils zu MZP 1 dar. Die Kovarianz von Random Intercept und Random Slope erlaubt Aussagen darüber, inwiefern Unterschiede in den Anfangswerten der Personen mit unterschiedlichen Entwicklungsverläufen über die Zeit assoziiert sind.

Für die Untersuchung von längsschnittlichen Daten bieten MEA im Vergleich zu Varianzanalysen mit Messwiederholung mehrere Vorteile (Heck et al. 2014). Zum einen können in Random Koeffizient-Modellen interindividuelle Unterschiede in den intraindividuellen Entwicklungsverläufen abgebildet werden (Laird und Ware 1982). Diese Variationen in den Entwicklungsparametern der Individuen können anhand weiterer Merkmale der Person (Level 2) erklärt werden. Ein weiterer Vorteil der MEA besteht darin, dass kein Ausschluss von Fällen mit fehlenden Werten an einzelnen Messzeitpunkten erfolgt (Anderson 2012; Heck et al. 2014). Der Einsatz von MEA ermöglicht die Untersuchung von Zusammenhängen zwischen der abhängigen Variable und anderen zeitvariierenden Kovariaten auf Level 1. Darüber hinaus können Cross-Level-Interaktionen modelliert werden, womit Effekte von Variablen der höheren Ebene auf die abhängige Variable oder auf eine Variable auf niedriger Ebene identifiziert werden können (Hox 2013).

In unserer Studie bildet die Zustimmung zu den alternativen Vorstellungen die abhängige Variable, deren Veränderung über die drei Messzeitpunkte anhand der Variable Zeit modelliert wird (Level 1); Level 2 bilden die einzelnen SuS bzw. deren Merkmale wie z. B. ihre Gruppenzugehörigkeit. Die drei Dimensionen des hypothesenbezogenen Schlussfolgerns wurden ebenfalls zu den drei Messzeitpunkten er- hoben, sodass sie als zeitvariierende Kovariaten auf Level 1 fungieren.

Zur Untersuchung der ersten Fragestellung bzgl. des Entwicklungsverlaufs der Zustimmung zu alternativen Vorstellungen wurden Random-Intercept-Random-SlopeModelle berechnet (s. Anderson, 2012). Zur Beschreibung der Entwicklungsverläufe wurden ein lineares Modell (Modell 1) mit der unabhängigen Variable Zeit (Level 1; $0=$ Prä, 1 = Post, 2 = Follow-Up) und ein kurvilineares Modell (Modell 2) mit den unabhängigen Variablen Zeit und Zeit ${ }^{2}$ als Polynomial berechnet und anhand der AIC- und BIC-Fitindizien miteinander verglichen. Der lineare Term stellt die Veränderungsrate für ein Zeitintervall dar und wird über die Zeit als konstant zwischen Prä und Post sowie Post und Follow-Up angesehen, wohingegen der quadratische Term die Veränderung in der Änderungsrate (verlangsamend oder beschleunigend) über die Zeit abbildet (Heck et al. 2014).

Zur Untersuchung der zweiten Fragestellung bzgl. des Zusammenhangs zwischen alternativen Vorstellungen und dem hypothesenbezogenen Schlussfolgern wurden die drei Dimensionen der Schlussfolgerungsfähigkeit als zeitvariierende z-standardisierte Kovariaten des Level 1 in die Analyse aufgenommen (Modell 3).

Zur Untersuchung der dritten Fragestellung bzgl. des Einflusses der Intervention auf die Veränderung von alternativen Vorstellungen wurden die Intervention als Level 2-Variable und die Cross-Level-Interaktionen zwischen $\mathrm{Zeit}^{2}$ und der Interventionsgruppe in die Analyse miteinbezogen (Modell 4).

Da für die drei Fragestellungen gerichtete Hypothesen vorliegen, wurde einseitig getestet und der $p$-Wert halbiert. Die Mehrebenenanalysen wurden in Anlehnung an Heck et al. (2014) mit der Software SPSS durchgeführt.

Tab. 1 Stichprobengröße $(N)$, Mittelwerte $(M)$ und Standardabweichungen $(S D)$ der Zustimmung zu alternativen Vorstellungen und der drei Dimensionen des hypothesenbezogenen Schlussfolgerns zu den drei Messzeitpunkten Prä (vor dem Unterricht), Post (nach dem Unterricht) und Follow-Up (12-14 Wochen nach dem Unterricht) für beide Interventionsgruppen zusammen sowie einzeln

\begin{tabular}{|c|c|c|c|c|c|c|c|c|c|}
\hline & & \multicolumn{2}{|c|}{$\begin{array}{l}\text { Zustimmung } \\
\text { zu alternativen } \\
\text { Vorstellungen } \\
\end{array}$} & \multicolumn{2}{|c|}{$\begin{array}{l}\text { Schlussfolgern } \\
\text { - bekräftigende } \\
\text { Ereignisse } \\
\end{array}$} & \multicolumn{2}{|c|}{$\begin{array}{l}\text { Schlussfolgern } \\
\text { - widerlegende } \\
\text { Ereignisse } \\
\end{array}$} & \multicolumn{2}{|c|}{$\begin{array}{l}\text { Schlussfolgern } \\
\text { - irrelevante } \\
\text { Ereignisse } \\
\end{array}$} \\
\hline & & $N$ & $M(S D)$ & $N$ & $M(S D)$ & $N$ & $M(S D)$ & $N$ & $M(S D)$ \\
\hline \multirow[t]{3}{*}{$\overline{\text { Gesamt }}$} & Prä & 133 & $3,35(1,50)$ & 135 & $0,61(0,43)$ & 134 & $0,72(0,23)$ & 131 & $0,30(0,33)$ \\
\hline & Post & 132 & $2,18(2,06)$ & 128 & $0,79(0,35)$ & 126 & $0,74(0,26)$ & 126 & $0,64(0,36)$ \\
\hline & Follow-Up & 125 & $2,29(1,97)$ & 135 & $0,82(0,33)$ & 134 & $0,77(0,29)$ & 130 & $0,65(0,37)$ \\
\hline \multirow[t]{3}{*}{ EG } & Prä & 68 & $3,37(1,44)$ & 71 & $0,63(0,41)$ & 71 & $0,71(0,25)$ & 69 & $0,27(0,29)$ \\
\hline & Post & 68 & $2,28(2,10)$ & 66 & $0,84(0,28)$ & 65 & $0,75(0,26)$ & 65 & $0,72(0,32)$ \\
\hline & Follow-Up & 62 & $2,01(1,85)$ & 68 & $0,82(0,34)$ & 67 & $0,75(0,30)$ & 65 & $0,71(0,35)$ \\
\hline \multirow[t]{3}{*}{$\mathrm{KG}$} & Prä & 65 & $3,34(1,56)$ & 64 & $0,59(0,44)$ & 63 & $0,73(0,20)$ & 62 & $0,34(0,38)$ \\
\hline & Post & 64 & $2,08(2,03)$ & 62 & $0,74(0,40)$ & 61 & $0,73(0,27)$ & 61 & $0,55(0,38)$ \\
\hline & Follow-Up & 63 & $2,56(2,05)$ & 67 & $0,84(0,30)$ & 67 & $0,80(0,27)$ & 65 & $0,60(0,39)$ \\
\hline
\end{tabular}

Werte der adäquaten Ereignisevaluationen sind als Anteil vom erreichbaren Maximalwert 1 angegeben

Die Stichprobengröße variiert je nach Anwesenheit der SuS zu den verschiedenen MZP 


\section{Ergebnisse}

\section{Deskriptive Statistiken}

Die deskriptiven Statistiken bzgl. der Zustimmung zu den präsentierten alternativen Vorstellungen und der drei Dimensionen des hypothesenbezogenen Schlussfolgerns sind für die drei Messzeitpunkte insgesamt und getrennt nach Gruppe in Tab. 1 dargestellt. Sie zeigen eine Abnahme der alternativen Vorstellungen im Laufe der Zeit. Während im Prätest noch im Mittel 3,35 alternativen Vorstellungen zugestimmt wurde, gibt es im Posttest im Mittel eine Zustimmung bei 2,18 alternativen Vorstellungen. Zum Follow-UpZeitpunkt zeigt sich im Vergleich zum Posttest ein geringfügiger Anstieg der Zustimmung, der Wert bleibt jedoch unter dem des Präzeitpunktes. In Bezug auf den Vergleich zwischen EG und KG ist zu erkennen, dass sich der Wiederanstieg der alternativen Vorstellungen nach dem Unterricht auf die KG beschränkt. Tab. 1 ist außerdem zu entnehmen, dass bei allen drei Dimensionen des hypothesenbezogenen Schlussfolgerns die adäquate Ereignisevaluation zunimmt.
Unterschiede zwischen den Gruppen zeigen sich insbesondere im Entwicklungsverlauf der irrelevanten Ereignisevaluationen; in der EG gibt es diesbezüglich eine stärkere Zunahme an adäquaten Schlussfolgerungen.

\section{Entwicklungsverläufe der Zustimmung zu alternativen Vorstellungen (erste Fragestellung)}

Der Vergleich zwischen dem Modell mit der Zeit als linearem Prädiktor (Modell 1) und dem kurvilinearen Modell (Modell 2) anhand der AIC- und BIC-Fitindizien zeigt eine bessere Passung des kurvilinearen Modells zu den Daten (s. Tab. 2). Die Fixed-Effekte für den Intercept in Modell 2 zeigen an, dass die SuS vor dem Unterricht durchschnittlich 3,35 von 8 alternativen Vorstellungen zugestimmt haben, wobei der Random-Effekt für den Intercept $\left(\mathrm{r}_{0}\right)$ nicht signifikant ist und auf eine geringe Heterogenität der Zustimmung zu alternativen Vorstellungen hindeutet. Den FixedEffekten der Zeitvariablen $\beta_{10}$ und $\beta_{20}$ ist zu entnehmen, dass die durchschnittliche Zustimmung zu den alternativen Vorstellungen insgesamt signifikant abnimmt $\left(\beta_{10}=-1,79\right.$;

Tab. 2 Modellvergleich zum Entwicklungsverlauf der Zustimmung zu alternativen Vorstellungen (Modell 1 und Modell 2), zum Zusammenhang mit dem hypothesenbezogenen Schlussfolgern (Evaluation von bekräftigenden, widerlegenden und irrelevanten Ereignissen in Modell 3) und zum Einfluss der Gruppenzugehörigkeit auf die Entwicklung von alternativen Vorstellungen (Modell 4)

\begin{tabular}{|c|c|c|c|c|}
\hline Modell & Modell 1 & Modell 2 & Modell 3 & Modell 4 \\
\hline Fixed Effekte & Koeffizient (SE) & Koeffizient (SE) & Koeffizient (SE) & Koeffizient (SE) \\
\hline Intercept $\beta_{00}$ & $\begin{array}{l}3,20 * * * \\
(0,12)\end{array}$ & $\begin{array}{l}3,35^{* * *} \\
(0,13)\end{array}$ & $\begin{array}{l}3,14 * * * \\
(0,15)\end{array}$ & $\begin{array}{l}3,21 * * * \\
(0,19)\end{array}$ \\
\hline Zeit $\beta_{10}$ & $\begin{array}{l}-0,45^{* * *} \\
(0,10)\end{array}$ & $\begin{array}{l}-1,79 * * * \\
(0,35)\end{array}$ & $\begin{array}{l}-1,55^{* * *} \\
(0,40)\end{array}$ & $\begin{array}{l}-1,55^{* * * *} \\
(0,41)\end{array}$ \\
\hline $\mathrm{Zeit}^{2} \beta_{20}$ & - & $\begin{array}{l}0,64 * * * \\
(0,19)\end{array}$ & $\begin{array}{l}0,62 * * * \\
(0,18)\end{array}$ & $\begin{array}{l}0,52 * * \\
(0,19)\end{array}$ \\
\hline Bekräftigend & - & - & $\begin{array}{l}-0,14 \\
(0,10)\end{array}$ & $\begin{array}{l}-0,14 \\
(0,10)\end{array}$ \\
\hline Widerlegend & - & - & $\begin{array}{l}-0,27 * * \\
(0,10)\end{array}$ & $\begin{array}{l}-0,28 * * \\
(0,10)\end{array}$ \\
\hline Irrelevant & - & - & $\begin{array}{l}-0,25^{*} \\
(0,11)\end{array}$ & $\begin{array}{l}-0,24^{*} \\
(0,11)\end{array}$ \\
\hline $\begin{array}{l}\text { Gruppe } \\
(0=\mathrm{EG}, 1=\mathrm{KG})\end{array}$ & - & - & - & $\begin{array}{l}-0,14 \\
(0,24)\end{array}$ \\
\hline $\begin{array}{l}\text { Gruppe*Zeit }{ }^{2} \\
(0=\mathrm{EG}, 1=\mathrm{KG})\end{array}$ & - & - & - & $\begin{array}{l}0,19 * \\
(0,10)\end{array}$ \\
\hline Random Effekte & $\begin{array}{l}\text { (Ko)Varianz } \\
(S E)\end{array}$ & $\begin{array}{l}(\text { Ko }) \text { Varianz } \\
(S E)\end{array}$ & $\begin{array}{l}\text { (Ko) Varianz } \\
(S E)\end{array}$ & $\begin{array}{l}\text { (Ko)Varianz } \\
(S E)\end{array}$ \\
\hline Intercept $\mathrm{r}_{0}$ & $\begin{array}{l}0,57 \\
(0,52)\end{array}$ & $\begin{array}{l}0,73 \\
(0,48)\end{array}$ & $\begin{array}{l}0,32 \\
(0,55)\end{array}$ & $\begin{array}{l}0,33 \\
(0,55)\end{array}$ \\
\hline Slope time $\mathrm{r}_{1}$ & $\begin{array}{l}0,90 * * * \\
(0,20)\end{array}$ & $\begin{array}{l}0,95^{* * *} \\
(0,20)\end{array}$ & $\begin{array}{l}0,97 * * \\
(0,33)\end{array}$ & $\begin{array}{l}0,96 * * * \\
(0,23)\end{array}$ \\
\hline Slope*Intercept $r_{2}$ & $\begin{array}{l}-0,08 \\
(0,29)\end{array}$ & $\begin{array}{l}-0,16 \\
(0,27)\end{array}$ & $\begin{array}{l}0,19 \\
(0,32)\end{array}$ & $\begin{array}{l}0,18 \\
(0,31)\end{array}$ \\
\hline \multicolumn{5}{|l|}{ Modellgüte } \\
\hline AIC & 1559,72 & 1546,07 & 1396,51 & 1396,82 \\
\hline $\mathrm{BIC}$ & 1583,48 & 1569,82 & 1419,60 & 1419,88 \\
\hline
\end{tabular}

$* * * p<0,001 ; * * p<0,01 ; * p<0,05$ 
$p<0,001)$, die Veränderung über die Zeit jedoch nicht linear absteigend ist, sondern sich verlangsamt bzw. mit der Zeit wieder geringfügig ansteigt, was sich in dem positiven signifikanten quadratischen Term der Variable widerspiegelt $\left(\beta_{20}=0,64 ; p<0,001\right)$. Die Entwicklungsverläufe der alternativen Vorstellungen über die Zeit weisen eine signifikante Heterogenität ( $\left.\mathrm{r}_{1}\right)$ auf (vgl. Tab. 2).

\section{Zusammenhang zwischen dem hypothesenbezogenen Schlussfolgern und alternativen Vorstellungen (zweite Fragestellung)}

Die Ergebnisse des weiteren Mehrebenenmodells mit Aufnahme der drei Dimensionen des hypothesenbezogenen Schlussfolgerns (s. Modell 3 in Tab. 2) zeigen negative signifikante Zusammenhänge zwischen der Zustimmung zu alternativen Vorstellungen und der Evaluation von widerlegenden Ereignissen sowie von irrelevanten Ereignissen. Nimmt die Schlussfolgerungsfähigkeit bei der Evaluation von widerlegenden Ereignissen (/von irrelevanten Ereignissen) um eine Standardabweichung zu, so geht die Anzahl an alternativen Vorstellungen um 0,27 (/0,25) zurück. Es zeigt sich ein ebenfalls negativer, aber nicht signifikanter Zusammenhang zwischen der Evaluation von bestätigenden Ereignissen und der Zustimmung zu alternativen Vorstellungen.

\section{Unterschiede im Entwicklungsverlauf der alternativen Vorstellungen in Abhängigkeit von der Interventionsgruppe (dritte Fragestellung)}

Die Ergebnisse der Cross-Level-Interaktionen mit der Gruppe als zusätzlichem Faktor auf Level 2 und dem quadrierten Term der Zeitvariable (s. Modell 4 in Tab. 2) zeigen weiterhin eine signifikante kurvilineare Veränderung über

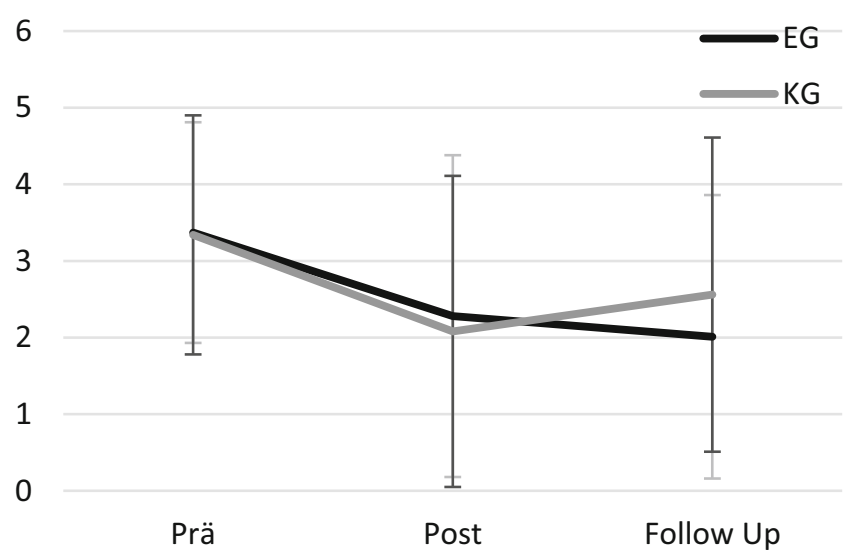

Abb. 1 Veränderung der alternativen Vorstellungen über die Zeit getrennt nach Experimentalgruppe $(E G)$ und Kontrollgruppe $(K G)$ (Die $\mathrm{y}$-Achse stellt die durchschnittliche Anzahl alternativer Vorstellungen dar) die Zeit, signifikante Zusammenhänge zwischen den alternativen Vorstellungen und den beiden Schlussfolgerungsdimensionen widerlegend und irrelevant und des Weiteren wie angenommen eine positive signifikante Interaktion der Gruppe mit der Zeit ${ }^{2}$-Variable. Unter Konstanthaltung aller weiteren Prädiktoren weist die KG im Vergleich zur EG eine um 0,19 höhere Verlangsamungsrate bei der Abnahme der alternativen Vorstellungen über die Zeit auf. Die Veränderung der alternativen Vorstellungen über die Zeit für beide Gruppen ist in Abb. 1 veranschaulicht.

\section{Diskussion}

\section{Zentrale Befunde}

In der vorliegenden Studie wurde die Veränderung der alternativen Vorstellungen über die Zeit (Hypothese 1), der Zusammenhang zwischen dem hypothesenbezogenen Schlussfolgern und alternativen Vorstellungen in einem inquiryorientierten Unterricht (Hypothese 2) und die Effekte einer Förderung des hypothesenbezogenen Schlussfolgerns auf den Entwicklungsverlauf der alternativen Vorstellungen (Hypothese 3) untersucht.

Die Ergebnisse zu Hypothese 1 zeigen, dass hypothesenkonform ein signifikanter Rückgang der Zustimmung zu den alternativen Vorstellungen von vor dem Unterricht $\mathrm{zu}$ unmittelbar nach dem Unterricht vorliegt. Im Follow-UpTest, 12-14 Wochen nach Abschluss des Unterrichts, stieg die Zustimmung zu den alternativen Vorstellungen wieder leicht an. Nach wie vor bestehen einige alternative Vorstellungen auch noch nach dem Unterricht bei den SuS. Damit bekräftigt das vorliegende Ergebnis die Befunde bisheriger Studien, dass Vorstellungen häufig tief verankert und damit nur schwer veränderbar sind (Duit 1995). Die Befunde der vorliegenden Studie sprechen auch dafür, dass sich Vorstellungsänderungen nicht abrupt vollziehen, sondern vielmehr in langwierigen Prozessen verlaufen (Limón 2001). Die Veränderung der alternativen Vorstellungen ist daher auch vor dem Hintergrund der Unterrichtsdauer zu betrachten. Die SuS haben sich drei Schulstunden lang mit der Überprüfung von alternativen Vorstellungen auseinandergesetzt, was der Möglichkeit zur Veränderung von alternativen Vorstellungen, die sich über Jahre hinweg aufgebaut haben, vermutlich nicht hinreichend gerecht werden kann. Wenn auch nicht in vollem Ausmaß, so findet doch eine Veränderung der alternativen Vorstellungen statt, welche auch noch einige Wochen nach dem Unterricht vorzufinden ist. Eine mögliche Erklärung hierfür ist, dass es für die SuS einen bedeutsamen kognitiven Konflikt gegeben haben könnte (Limón 2001), sodass sich die Überzeugung bzgl. der alternativen Vorstellungen nachhaltig verändert haben müsste. Die Zunahme der Zustimmung zu alternativen Vor- 
stellungen im Follow-Up-Test lässt vermuten, dass manche Vorstellungen so tief verankert sind, dass sie nach einiger Zeit wieder auftreten und ggf. parallel zum wissenschaftlich anerkannten Konzept bestehen. Insgesamt kann die Hypothese eines nicht vollständigen Rückgangs der Zustimmung $\mathrm{zu}$ alternativen Vorstellungen bekräftigt werden.

Bei der Untersuchung des Zusammenhangs zwischen dem hypothesenbezogenen Schlussfolgern und den alternativen Vorstellungen (Hypothese 2) zeigen sich unterschiedliche Ergebnisse in Abhängigkeit von den verschiedenen Dimensionen der Schlussfolgerungsfähigkeit bei konditionalen Aussagen. Bei einer höheren Ausprägung der Fähigkeit, widerlegende und irrelevante Ereignisse adäquat $\mathrm{zu}$ evaluieren, besteht hypothesenkonform eine geringere $\mathrm{Zu}$ stimmung zu den alternativen Vorstellungen. Bei der Fähigkeit, bekräftigende Ereignisse adäquat zu evaluieren, zeigte sich allerdings kein Zusammenhang mit den alternativen Vorstellungen.

Eine mögliche Erklärung des Befunds, dass ein Zusammenhang zwischen der Schlussfolgerungsfähigkeit und den alternativen Vorstellungen in Bezug auf die Evaluation widerlegender und irrelevanter Ereignisse, nicht jedoch in Bezug auf bekräftigende Ereignisse besteht, liegt vermutlich in der Natur der bekräftigenden Ereignisse begründet. Bei einem bekräftigenden Ereignis besteht kein Anlass zur Entstehung von Unzufriedenheit mit dem vorhandenen Konzept, sodass folglich weder ein kognitiver Konflikt entstehen kann noch die Notwendigkeit einer Veränderung der alternativen Vorstellung besteht. Das widerlegende Ereignis hingegen ist ausschlaggebend für das Aufkommen von Unzufriedenheit mit der vorhandenen alternativen Vorstellung. Daher kann vermutet werden, dass Lernende mit gering ausgeprägter Schlussfolgerungsfähigkeit dazu neigen, widerlegende Ereignisse als bekräftigend oder zumindest als irrelevant zu evaluieren, um an ihrem Glauben an die alternative Vorstellung festhalten zu können. Weniger offensichtlich ist die Erklärung des Zusammenhangs zwischen alternativen Vorstellungen und der Evaluation von irrelevanten Ereignissen. Eine mögliche Erklärung könnte sein, dass irrelevante Ereignisse von Lernenden als Bekräftigung ihrer alternativen Vorstellungen herangezogen werden. Eine solche fehlerhafte Evaluation von irrelevanten Ereignissen würde zu einer weiteren unberechtigten Bestätigung der alternativen Vorstellung führen. Insbesondere wenn bei den SuS keine Einsicht in das Falsifikationsprinzip besteht (Moshman 1979), könnte der Zusammenhang zwischen alternativen Vorstellungen und der Fähigkeit zur Evaluation von irrelevanten Ereignissen auf diese Weise erklärt werden.

Weiterhin zeigen die Ergebnisse (Hypothese 3), dass der Einsatz von Scaffoldingmaßnahmen zur Förderung der Schlussfolgerungsfähigkeit anscheinend einen positiven Effekt auf den Entwicklungsverlauf der alternativen Vorstel- lungen hat. Im Verlauf der Zeit kehren die alternativen Vorstellungen in der KG stärker zurück als in der EG. Dieser längerfristige Rückgang von alternativen Vorstellungen beim Einsatz von Scaffoldingmaßnahmen steht in Konsens mit den Ergebnissen von Hardy et al. (2006). Eine mögliche Erklärung hierfür wäre, dass die Lernenden ohne eine zusätzliche Förderung der Schlussfolgerungsfähigkeit möglicherweise keinen Konflikt zwischen Evidenz und den eigenen Vorstellungen erfahren haben. Nach Vosniadou und Brewer (1992) kann es daher zu keiner Auflösung von Inkonsistenzen zwischen alternativen Vorstellungen und wissenschaftlicher Theorie kommen, sodass alternative Vorstellungen langfristig parallel zum neu erlangten Konzept bestehen bleiben könnten.

Insgesamt geben die Ergebnisse Anlass zu der Annahme, dass die Fähigkeit zum hypothesenbezogenen Schlussfolgern Vorstellungsänderungen begünstigen kann. Befunde vorangegangener Studien können daher mit den Ergebnissen der vorliegenden Studie für den Bereich des Schlussfolgerns bei konditionalen Aussagen bekräftigt werden (Kang et al. 2004; Liao und She 2009). Darüber hinaus konnte gezeigt werden, dass die bisherigen Befunde, welche sich auf SuS ab der siebten Klassenstufe beziehen, auch bereits für Kinder im Grundschulalter zuzutreffen scheinen.

Anhand der Anlage des Studiendesigns ist es nicht möglich, eine Aussage darüber zu treffen, ob die Ursache des Rückgangs der Zustimmung zu alternativen Vorstellungen in der Erzeugung eines kognitiven Konflikts beim Lernenden begründet liegt. Die Messung des kognitiven Konflikts selbst ist methodisch nur schwer umsetzbar (Hemmerich et al. 2016; Limón 2001), sodass in der vorliegenden Studie das Testinstrument auf die Messung der Vorstellungsänderung beschränkt war. So zeigen die vorliegenden Ergebnisse zunächst lediglich, dass höhere Werte beim hypothesenbezogenen Schlussfolgern mit einer geringeren Anzahl an zugestimmten alternativen Vorstellungen einhergehen. Dies gibt jedoch Anlass zu der Vermutung, dass die Schlussfolgerungsfähigkeit auch in einem Zusammenhang mit der Erzeugung eines bedeutsamen kognitiven Konfliktes steht (Limón 2001).

Zwar konnte ein Zusammenhang zwischen den alternativen Vorstellungen und der Schlussfolgerungsfähigkeit gezeigt werden; es ist jedoch zu bedenken, dass die Veränderung von Vorstellungen ein komplexer Vorgang ist, der von vielen verschiedenen Faktoren abhängig ist (Leonard et al. 2015; Limón 2001). Wenn andere Faktoren, wie zum Beispiel die Motivation, im Lernprozess nicht gegeben sind, kann die Erzeugung eines kognitiven Konflikts trotz vorhandener Schlussfolgerungsfähigkeit verhindert werden. Dennoch scheint die Schlussfolgerungsfähigkeit ein wichtiger Faktor im Rahmen des Konfliktstrategieansatzes zu sein, welcher das Hervorrufen von Unzufriedenheit mit der alternativen Vorstellung erzeugen kann. 
Damit scheint die Schlussfolgerungsfähigkeit ein relevanter Faktor für fachliches Lernen in einem inquiry-orientiertem Unterricht zu sein. Da jedoch sowohl bei Grundschulkindern, aber auch bei älteren SuS (Barrouillet et al. 2008; Gauffroy und Barrouillet 2011), häufig inadäquate Schlussfolgerungen bei der Überprüfung von konditionalen Aussagen getroffen werden, erweist sich eine unterrichtliche Förderung des hypothesenbezogenen Schlussfolgerns als erforderlich, um im Rahmen einer naturwissenschaftlichen Grundbildung sowohl prozessbezogene als auch fachinhaltliche Kompetenzen zu fördern.

\section{Limitationen}

Die Ergebnisse der vorliegenden Studie sollten vor dem Hintergrund gewisser methodischer Einschränkungen betrachtet werden. Zum einen ist zu berücksichtigen, dass bei der genutzten Methode der Erfassung von alternativen Vorstellungen lediglich gezeigt werden kann, dass es zu einer Reduktion der alternativen Vorstellungen im Laufe der Zeit kommt. Es wird nicht ersichtlich, ob es sich bei den Vorstellungen der SuS um deep oder um current constructions (Niedderer und Schecker 1992) handelt, sodass keine Aussage darüber getroffen werden kann, ob auch eine Veränderung der tief verankerten alternativen Vorstellungen durch den Unterricht gelungen ist.

Ein weiterer zu betrachtender Aspekt bei der Interpretation der Ergebnisse ist die Anlage der Untersuchung. Vor dem Hintergrund, dass die Bereitschaft, alternative Vorstellungen aufzugeben, steigt, wenn ein neues Konzept angeboten wird (Hemmerich et al. 2016), wurde der Test zur Erfassung der Zustimmung zu alternativen Vorstellungen erst nach Abschluss des gesamten Unterrichts eingesetzt, während der Schlussfolgerungstest bereits nach Abschluss der Sequenzen zur Überprüfung von alternativen Vorstellungen eingesetzt wurde. Da in den letzten drei Sequenzen jedoch der Aufbau des wissenschaftlich adäquaten Konzepts im Vordergrund stand, ist kein weiterer Einfluss einer Auseinandersetzung mit alternativen Vorstellungen zwischen den beiden Postzeitpunkten anzunehmen.

Weiterhin ist zu beachten, dass die mit einer mehrstufigen Skala erfasste Zustimmung zu alternativen Vorstellungen letztlich dichotom ausgewertet wurde. Dies ermöglichte eine kompaktere Darstellung der Ergebnisse, während eine einzelne Berücksichtigung aller Antwortoptionen den vermutlich graduell verlaufenden Prozess von Vorstellungsänderungen hätte genauer abbilden können.

Schließlich ist anzumerken, dass in den vorliegenden MEA die Zugehörigkeit der Kinder zu Klassen nicht in den Analysen mitberücksichtigt wurde. Die Berücksichtigung dieser dritten Ebene würde die Komplexität der Modelle erhöhen und eine weitaus größere Stichprobe als in der vorliegenden Studie vorhanden erfordern (Hox 2013), wes- halb die Auswertungen im Rahmen des 2-Level-Modells erfolgten.

\section{Ausblick}

In der vorliegenden Studie wurde der Zusammenhang zwischen dem hypothesenbezogenen Schlussfolgern und alternativen Vorstellungen untersucht. Hieran knüpfen unmittelbar zwei weitere Forschungsinteressen an.

Eine weitere wichtige Fähigkeit, um eigene alternative Vorstellungen als solche zu erkennen, ist die Einsicht in das Falsifikationsprinzip. Nur wenn vom Lernenden erkannt wird, dass das eine erkannte diskrepante Ereignis ausreicht, um eine Vermutung endgültig zu widerlegen (Moshman 1979), kann Unzufriedenheit mit den vorhandenen alternativen Vorstellungen entstehen und ein bedeutsamer kognitiver Konflikt erzeugt werden. Daher wäre zukünftig zu untersuchen, ob neben der Fähigkeit, Ereignisse adäquat zu evaluieren, zusätzlich die Einsicht in das Falsifikationsprinzip zur Veränderung von Vorstellungen erforderlich ist.

Anknüpfend an die vorliegende Untersuchung der Veränderung von alternativen Vorstellungen betrifft ein weiteres Forschungsvorhaben den Aufbau des wissenschaftlich adäquaten Konzepts. Letztendlich sollen die Präsentation von diskrepanten Ereignissen sowie die Veränderung von alternativen Vorstellungen zu einem verständnisvollen Aufbau des wissenschaftlich anerkannten Konzepts führen. Ausgehend von Befunden von Hemmerich et al. (2016) kann angenommen werden, dass es einen solchen Zusammenhang zwischen dem Rückgang der Überzeugung bzgl. alternativer Vorstellungen und dem Aufbau des wissenschaftlichen Konzepts gibt. Die Untersuchung dieses Zusammenhangs könnte einen Erkenntnisgewinn für die Wirksamkeit der Konfliktstrategie und damit relevante Implikationen für die schulische Praxis hervorbringen.

Förderung Diese Veröffentlichung wurde ermöglicht durch eine Sachbeihilfe der Deutschen Forschungsgemeinschaft (Kennz.: MO942/6-1).

Funding Open Access funding provided by Projekt DEAL.

Open Access Dieser Artikel wird unter der Creative Commons Namensnennung 4.0 International Lizenz veröffentlicht, welche die Nutzung, Vervielfältigung, Bearbeitung, Verbreitung und Wiedergabe in jeglichem Medium und Format erlaubt, sofern Sie den/die ursprünglichen Autor(en) und die Quelle ordnungsgemäß nennen, einen Link zur Creative Commons Lizenz beifügen und angeben, ob Änderungen vorgenommen wurden.

Die in diesem Artikel enthaltenen Bilder und sonstiges Drittmaterial unterliegen ebenfalls der genannten Creative Commons Lizenz, sofern sich aus der Abbildungslegende nichts anderes ergibt. Sofern das betreffende Material nicht unter der genannten Creative Commons Lizenz steht und die betreffende Handlung nicht nach gesetzlichen Vorschriften erlaubt ist, ist für die oben aufgeführten Weiterverwendungen des Materials die Einwilligung des jeweiligen Rechteinhabers einzuholen. 
Weitere Details zur Lizenz entnehmen Sie bitte der Lizenzinformation auf http://creativecommons.org/licenses/by/4.0/deed.de.

\section{Literatur}

Adamina, M., Kübler, M., Kalcsis, K., Bietenhard, S., \& Engeli, E. (2018). Vorstellungen von Schülerinnen und Schülern zu Themen des Sachunterrichts und des Fachbereichs Natur, Mensch, Gesellschaft - Einführung. In M. Adamina, M. Kübler, K. Kalcsis, S. Bietenhard \& E. Engeli (Hrsg.), ,Wie ich mir das denke und vorstelle... “. Vorstellungen von Schülerinnen und Schülern zu Lerngegenständen des Sachunterrichts und des Fachbereichs Natur, Mensch, Gesellschaft (S. 7-20). Bad Heilbrunn: Klinkhardt.

Alfieri, L., Brooks, P. J., Aldrich, N.J., \& Tenenbaum, H.R. (2011). Does discovery-based instruction enhance learning? Journal of Educational Psychology, 103, 1-18.

Anderson, D. R. (2012). Hierarchical linear modeling (HLM): an introduction to key concepts within cross-sectional and growth modeling frameworks. Behavioral research and teaching. Oregon: University of Oregon.

Barrouillet, P., Gauffroy, C., \& Lecas, J.-F. (2008). Mental models and the suppositional account of conditionals. Psychological Review, $115,760-771$.

Bybee, R. (2002). Scientific Literacy - Mythos oder Realität? In W. Gräber, P. Nentwig, T. Koballa \& R. Evans (Hrsg.), Scientific Literacy. Der Beitrag der Naturwissenschaften zur Allgemeinen Bildung (S. 21-43). Wiesbaden: VS.

Caravita, S. (2001). A re-framed conceptual change theory? Learning and Instruction, 11, 421-429.

Caravita, S., \& Halldén, O. (1994). Re-framing the problem of conceptual change. Learning and Instruction, 4, 89-111.

Carey, S. (2000). Science education as conceptual change. Journal of Applied Developmental Psychology, 21, 13-19.

Chinn, C., \& Brewer, W. (1993). The role of anomalous data in knowledge acquisition: a theoretical framework and implications for science instruction. Review of Educational Research, 63, 1-49.

Chinn, C. A., \& Brewer, W.F. (1998). An empirical test of a taxonomy of responses to anomalous data in science. Journal of Research in Science Teaching, 35, 623-654.

Decristan, J., Hondrich, A.L., Büttner, G., Hertel, S., Klieme, E., \& Kunter, M. (2015). Impact of additional guidance in science education on primary students' conceptual understanding. The Journal of Educational Research, 108, 358-370.

Duit, R. (1995). Zur Rolle der konstruktivistischen Sichtweise in der naturwissenschaftsdidaktischen Lehr- und Lernforschung. Zeitschrift für Pädagogik, 41, 905-923.

Duit, R., \& Treagust, D. (2003). Conceptual change: a powerful framework for improving science teaching and learning. International Journal of Science Education, 25, 671-688.

Dunn, T. J., Baguley, T., \& Brunsden, V. (2014). From alpha to omega: a practical solution to the pervasive problem of internal consistency estimation. British Journal of Psychology, 105, 399-412.

Edelsbrunner, P. A., Schalk, L., Schumacher, R., \& Stern, E. (2018). Variable control and conceptual change. A large-scale quantitative study in elementary school. Learning and Individual Differences, $66,38-53$.

Furtak, E. M., Seidel, T., Iverson, H., \& Briggs, D.C. (2012). Experimental and quasi-experimental studies of inquiry-based science teaching. A meta-analysis. Review of Educational Research, 82, 300-329.

Gauffroy, C., \& Barrouillet, P. (2009). Heuristic and analytic processes in mental models for conditionals: an integrative developmental theory. Developmental Review, 29, 249-282.

Gauffroy, C., \& Barrouillet, P. (2011). The primacy of thinking about possibilities in the development of reasoning. Developmental Psychology, 47, 1000-1011.
GDSU, Gesellschaft für Didaktik des Sachunterrichts (Hrsg.). (2013). Perspektivrahmen Sachunterricht. Bad Heilbrunn: Klinkhardt.

Grimm, H., Robisch, C., \& Möller, K. (2018). Förderung hypothesenbezogener Schlussfolgerungen im naturwissenschaftlichen Sachunterricht durch gezieltes Scaffolding - Gelingt dies unter Feldbedingungen? Zeitschrift für Grundschulforschung, 11, 349-363.

Gropengießer, H., \& Marohn, A. (2018). Schülervorstellungen und Conceptual Change. In D. Krüger, I. Parchmann \& H. Schecker (Hrsg.), Theorien in der naturwissenschaftsdidaktischen Forschung (S. 49-67). Berlin: Springer.

Handley, S. J., Capon, A., Beveridge, M., Dennis, I., \& Evans, J. S. B. (2004). Working memory, inhibitory control and the development of children's reasoning. Thinking \& Reasoning, 10, 175-195.

Hardy, I., \& Meschede, N. (2018). Schülervorstellungen - lernund entwicklungspsychologische Grundlagen. In M. Adamina, M. Kübler, K. Kalcsis, S. Bietenhard \& E. Engeli (Hrsg.), „Wie ich mir das denke und vorstelle... “. Vorstellungen von Schülerinnen und Schülern zu Lerngegenständen des Sachunterrichts und des Fachbereichs Natur, Mensch, Gesellschaft (S. 7-20). Bad Heilbrunn: Klinkhardt.

Hardy, I., Jonen, A., Möller, K., \& Stern, E. (2006). Effects of instructional support within constructivist learning environments for elementary school students' understanding of "floating and sinking.". Journal of Educational Psychology, 98, 307-326.

Heck, R.H., Thomas, S. L., \& Tabata, L. N. (2014). Multilevel and longitudinal modeling with IBM SPSS (2. Aufl.). New York: Taylor $\&$ Francis.

Hemmerich, J. A., van Voorhis, K., \& Wiley, J. (2016). Anomalous evidence, confidence change, and theory change. Cognitive Science, 40, 1534-1560.

Hox, J. J. (2013). Multilevel regression and multilevel structural equation modeling. In T. D. Little (Hrsg.), Oxford library of psychology. The Oxford handbook of quantitative methods: statistical analysis (S. 281-294). New York: Oxford University Press.

Johnson-Laird, P. N., \& Byrne, R. M. J. (2002). Conditionals: a theory of meaning, pragmatics, and inference. Psychological Review, 109, 646-678.

Kang, H., Scharmann, L.C., Kang, S., \& Noh, T. (2010). Cognitive conflict and situational interest as factors influencing conceptual change. International Journal of Environmental and Science Education, 5, 383-405.

Kang, S., Scharmann, L.C., \& Noh, T. (2004). Reexamining the role of cognitive conflict in science concept learning. Research in Science Education, 34, 71-96.

Klauer, K.C., Stegmaier, R., \& Meiser, T. (1997). Working memory involvement in propositional and spatial reasoning. Thinking \& Reasoning, 3, 9-47.

Kleickmann, T. (2012). Kognitiv aktivieren und inhaltlich strukturieren im naturwissenschaftlichen Sachunterricht. Handreichungen des Programms SINUS an Grundschulen. Kiel: IPN Leibniz-Institut für die Pädagogik der Naturwissenschaften und Mathematik an der Universität Kiel.

Laird, N.M., \& Ware, J.H. (1982). Random-effects models for longitudinal data. Biometrics, 38, 963-974.

Lawson, A.E. (1978). The development and validation of a classroom test of formal reasoning. Journal of Research in Science Teaching, $15,11-24$

Lawson, A. E. (2003). The neurological basis of learning, development and discovery. London: Kluwer Academic.

Lawson, A.E. (2009). Basic inferences of scientific reasoning, argumentation, and discovery. Science Education, 94, 336-364.

Lawson, A.E., \& Weser, J. (1990). The rejection of nonscientific beliefs about life: effects of instruction and reasoning skills. Journal of Research in Science Teaching, 27, 589-606.

Lee, C.-Q., \& She, H.-C. (2010). Facilitating students' conceptual change and scientific reasoning involving the unit of combustion. Research in Science Education, 40, 479-504. 
Lee, G., \& Byun, T. (2012). An explanation for the difficulty of leading conceptual change using a counterintuitive demonstration: the relationship between cognitive conflict and responses. Research in Science Education, 42, 943-965.

Leonard, M. J., Kalinowski, S.T., \& Taper, M.L. (2015). More than ideas themselves: influence of student attributes in conceptual change. The Researcher, 27, 34-42.

Liao, Y.-W., \& She, H.-C. (2009). Enhancing eight grade students' scientific conceptual change and scientific reasoning through a webbased learning program. Educational Technology \& Society, 12, 228-240.

Limón, M. (2001). On the cognitive conflict as an instructional strategy for conceptual change: a critical appraisal. Learning and Instruction, 11, 357-380.

Lin, J.-W., Yen, M.-H., Liang, J.-C., Chiu, M.-H., \& Guo, C.-J. (2016). Examining the factors that influence students' science learning processes and their learning outcomes: 30 years of conceptual change research. Eurasia Journal of Mathematics, Science and Technology Education, 12, 2617-2646.

Minner, D. D., Levy, A. J., \& Century, J. (2010). Inquiry-based science instruction-what is it and does it matter? Results from a research synthesis years 1984 to 2002. Journal of Research in Science Teaching, 47, 474-496.

Möller, K. (2018). Die Bedeutung von Schülervorstellungen für das Lernen im Sachunterricht. In M. Adamina, M. Kübler, K. Kalcsis, S. Bietenhard \& E. Engeli (Hrsg.), ,, Wie ich mir das denke und vorstelle... “. Vorstellungen von Schülerinnen und Schülern zu Lerngegenständen des Sachunterrichts und des Fachbereichs Natur, Mensch, Gesellschaft (S. 35-50). Bad Heilbrunn: Klinkhardt.

Möller, K. (2019). Lernen von Naturwissenschaften heißt: Vorstellungen verändern. In P. Labudde \& S. Metzger (Hrsg.), Fachdidaktik Naturwissenschaft 1.-9. Schuljahr (S. 59-74). Stuttgart: Haupt.

Moshman, D. (1979). Development of formal hypothesis-testing ability. Developmental Psychology, 15, 104-112.

Niedderer, H., \& Schecker, H. (1992). Towards an explicit description of cognitive systems for research in physics learning. In R. Duit, F. Goldberg \& H. Niedderer (Hrsg.), Research in physics learning-theoretical issues and empirical studies (S. 74-98). Kiel: IPN.

Ohlsson, S. (2009). Resubsumption: a possible mechanism for conceptual change and belief revision. Educational Psychologist, 44, $20-40$.

van de Pol, J., Volman, M., \& Beishuizen, J. (2010). Scaffolding in teacher-student interaction. A decade of research. Educational Psychology Review, 22, 271-296.

Pollmeier, J., Tröbst, S., Hardy, I., Möller, K., Kleickmann, T., Jurecka, A., \& Schwippet, K. (2017). Science-P I: modeling con- ceptual understanding in primary school. In D. Leutner, J. Fleischer, J. Grünkorn \& E. Klieme (Hrsg.), Competence assessment in education. Research, models and instruments (S. 9-17). Cham: Springer.

Posner, G., Strike, K., Hewson, P., \& Gertzog, W. (1982). Accommodation of a scientific conception: toward a theory of conceptual change. Science Education, 66, 211-227.

Prenzel, M., Schöps, K., Rönnebeck, S., Senkbeil, M., Walter, O., Carstensen, C.H., \& Hammann, M. (2007). Naturwissenschaftliche Kompetenz im internationalen Vergleich. In PISA-Konsortium Deutschland (Hrsg.), PISA 2006, Die Ergebnisse der dritten internationalen Vergleichsstudie (S. 63-105). Münster: Waxmann.

Ramsburg, J.T., \& Ohlsson, S. (2016). Category change in the absence of cognitive conflict. Journal of Educational Psychology, 108, 98-113.

Robisch, C., Tröbst, S., \& Möller, K. (2014). Hypothesenbezogene Schlussfolgerungen im Grundschulalter fördern. Zeitschrift für Grundschulforschung, 2, 88-101.

Scott, P. H., Asoko, H. M., \& Driver, R. (1992). Teaching for conceptual change: a review of strategies. In R. Duit, F. Goldberg \& H. Niederer (Hrsg.), Research in physics learning: theoretical issues and empirical studies (S. 310-329). Kiel: IPN.

Smith, J. P., diSessa, A. A., \& Roschelle, J. (1993). Misconceptions reconceived: a constructivist analysis of knowledge in transition. The Journal of the Learning Sciences, 3, 115-116.

Thiel, S. (1987). Wie springt ein Ball? Grundschule, 1, 18-23.

Tröbst, S., Hardy, I., \& Möller, K. (2011). Die Förderung deduktiver Schlussfolgerungen bei Grundschulkindern in naturwissenschaftlichen Kontexten. Unterrichtswissenschaft, 39, 7-20.

Vosniadou, S., \& Brewer, W.F. (1992). Mental models of the earth: a study of conceptual change in childhood. Cognitive Psychology, $24,35-585$.

Vosniadou, S., Ioannides, C., Dimitrakopoulou, A., \& Papademetriou, E. (2001). Designing learning environments to promote conceptual change in science. Learning and Instruction, 11, 381-419.

Wandersee, J.H., Mintzes, J. J., \& Novak, J. D. (1994). Research on alternative conceptions. In D. Gabel (Hrsg.), Handbook of research on science teaching and learning (S. 177-210). New York: Macmillan.

Wittwer, J., \& Renkl, A. (2008). Why instructional explanations often do not work: a framework for understanding the effectiveness of instructional explanations. Educational Psychologist, 43, 49-64.

Wodzinski, R. (2006). Lernschwierigkeiten erkennen - verständnisvolles Lernen fördern. Modulbeschreibungen des Programms Sinus an Grundschulen. Kiel: IPN. 